\title{
A new double-rebridging technique for linear polyethylene
}

\author{
Brian J. Banaszak and Juan J. de Pablo ${ }^{\text {a) }}$ \\ Department of Chemical Engineering, University of Wisconsin-Madison, Madison, Wisconsin 53706
}

(Received 20 February 2003; accepted 24 April 2003)

\begin{abstract}
A variable connectivity, double-rebridging Monte Carlo (MC) technique is developed for simulation of long chain molecules. The method changes the connectivity of inner segments of two chain molecules by making use of a recently proposed inner-chain rebridging scheme [Chen et al., J. Chem. Phys. 113, 11382 (2000)]. The new method yields results consistent with other molecular dynamics and MC methods, but it enhances considerably the rate of equilibration of chain end-to-end vectors for long molecules. The new method is tested for linear polyethylene melts at $600 \mathrm{~K}$. Polyethylene is modeled as linear 200 and 1000 carbon chains, respectively, using the NERD united-atom force-field (Nath, Escobedo, and de Pablo revised united-atom force field) [Nath et al., J. Chem. Phys. 108, 9905 (1998); Mol. Phys. 98, 231 (2000); J. Chem. Phys. 114, 3612 (2001)]. (C) 2003 American Institute of Physics. [DOI: 10.1063/1.1583673]
\end{abstract}

\section{INTRODUCTION}

Traditional Monte Carlo (MC) and molecular dynamics (MD) methods face severe limitations when simulating highly entangled polymer melts. Upon entanglement, the dynamics of polymer chains gradually crosses over from Rouse to reptation-type dynamics. This crossover slows down the relaxation of long-range modes considerably, thereby making them inaccessible to molecular dynamics simulations. Traditional MC simulations also face obstacles because conventional trial moves are performed on local scales.

To overcome these limitations, Theodorou and coworkers have proposed connectivity altering $\mathrm{MC}$ moves ${ }^{1-7}$ that improve the relaxation of long-range modes, namely the end-to-end autocorrelation function. In one such move, the connectivity of inner segments of a molecule is exchanged between two polymer chains, thereby resulting in a drastic change in the end-to-end vector in a single move. ${ }^{1}$ In this so-called "double-rebridging" technique, the reconstruction of the two chains is performed with trimers through solution of a geometric problem. ${ }^{2}$ Rigid bond lengths and bond angles are employed in this scheme. The acceptance of a typical double-rebridging move is extremely low, $\sim 0.002 \%$, but the effect is so dramatic that significant enhancement of the endto-end vector autocorrelation function is observed. Applications of the double-rebridging technique have been presented for linear polyethylene melts of 500 and 1000 carbons, respectively, at $450 \mathrm{~K}$ and $1 \mathrm{~atm}$.

In this work, an alternative double-rebridging move is proposed in which rebridging is conducted via the innerchain rebridging scheme of Chen et al. ${ }^{8}$ In this scheme, bond lengths and bonding angles are flexible. This technique is easily extendable to branched chain simulations and permits rebridging with as many segments as desired. In this frame-

\footnotetext{
a) Author to whom correspondence should be addressed. Electronic mail: depablo@engr.wisc.edu
}

work, the number of rebridging segments can be optimized for specific simulation conditions.

\section{SINGLE-REBRIDGING TECHNIQUE}

Before discussing the double-rebridging technique proposed here, it is instructive to review the single-rebridging scheme of Chen et al. ${ }^{8}$ for linear chains. In the singlerebridging scheme, a move is made to reconstruct the site positions of an inner section of a linear chain. An illustration of the proposed move is shown in Fig. 1.

Before a simulation involving this rebridging scheme is conducted, a presimulation is run in order to generate histograms for initial guess site-site separation probabilities. This presimulation can be an MC or MD simulation for an isolated chain, or a short simulation at system conditions.

Figure 2 illustrates the generation of site $i$ orientations for $i$ more than two sites away from chain closure. For $i>2, N_{\text {samp }}$ trial orientations for site $i$ are generated using an acceptance-rejection scheme. ${ }^{9,10}$ In this scheme, an orientation with a random direction on a sphere ${ }^{11}$ and a bond length distributed according to a harmonic spring ${ }^{10}$ are generated. This orientation is selected as a trial orientation according to the probability of the new bonded interactions introduced with the site (bond stretching, bond bending, and torsional interactions). ${ }^{9}$ One of the trial orientations is selected for site $i$ according to

$$
P\left(\mathbf{r}_{i}\right)=P_{\text {sep }}^{0}\left(r_{i, 0}\right) \exp \left(-\beta U_{i}^{\mathrm{ext}}\left(\mathbf{r}_{i}\right)\right) / w_{i},
$$

where

$$
w_{i}=\sum_{j=1}^{N_{\mathrm{samp}}} \exp \left(-\beta U_{i(j)}^{\mathrm{ext}}\left(\mathbf{r}_{i(j)}\right)\right) P_{\mathrm{sep}}^{0}\left(r_{i(j), 0}\right)
$$

where $P_{\text {sep }}^{0}\left(r_{i, 0}\right)$ is the "initial-guess" separation probability (from the presimulation) for sites separated by $i$ sites being at 


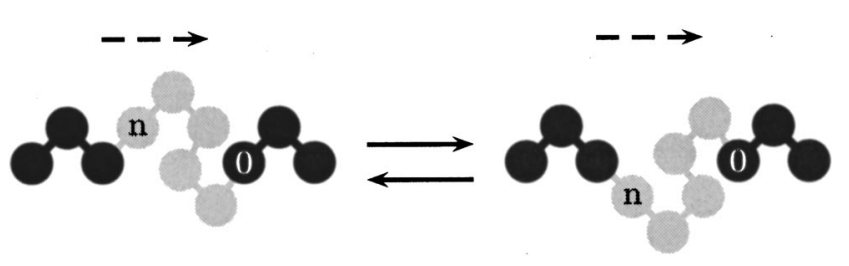

FIG. 1. Illustration of a single-rebridging move. The figure on the left is the old configuration and the figure on the right is the proposed new configuration. The dashed arrows indicate the order of bridge growth in terms of the site nomenclature of the figure.

a distance $r_{i, 0}$ apart, $\mathbf{r}_{i}$ is the position of site $i$, and $U_{i}^{\text {ext }}\left(\mathbf{r}_{i}\right)$ is the nonbonded energy associated with site $i$. The role of the additional site-site separation probability bias is to direct the generation of configurations favorable for final chain closure.

Figure 3 illustrates the generation of the second-to-last site before chain closure. In order to enhance chain closure, a stronger condition is desired for the acceptance criteria. For $i=2, N_{\text {samp }}$ trial orientations are generated in the same manner as for the $i>2$ case. In addition, the final two bond lengths, $b_{2,1}$ and $b_{1,0}$, are generated according to harmonic spring distributions. With the two bond lengths, the final bending angle, $\theta_{1}$, is also known. In addition, the selection of site $i=2$ restricts the location of site 1 to the circumference of the disk shown in Fig. 3. The final bending angle, $\theta_{1}$, and the Jacobian factor, $J\left(\mathbf{r}_{2}\right)$, for the crank-shaft rotation of site 1 are thus incorporated into the selection of site 2 according to

$$
P\left(\mathbf{r}_{2}\right)=\exp \left(-\beta U^{\text {ext }}\left(\mathbf{r}_{2}\right)\right) \exp \left(-\beta U^{\text {bend }}\left(\theta_{1}\right)\right) J\left(\mathbf{r}_{2}\right) / w_{2},
$$

where

$$
\begin{aligned}
w_{2}= & \sum_{j=1}^{N_{\text {samp }}} \exp \left(-\beta U^{\text {ext }}\left(\mathbf{r}_{2(j)}\right)\right) \\
& \times \exp \left(-\beta U^{\text {bend }}\left(\theta_{1(j)}\right)\right) J\left(\mathbf{r}_{2(j)}\right),
\end{aligned}
$$

and $J\left(\mathbf{r}_{2}\right)=1 /\left(b_{1,0} b_{2,1} r_{2,0}\right),{ }^{12,13}$ thereby taking into account the Jacobian for insertion of the last site on the disk, as shown in Fig. 3.

For $i=1$, a crank-shaft insertion is performed by randomly inserting site 1 onto the disk determined from the $i$ $=2$ site selection. One of the $N_{\text {samp }}$ sites is accepted according to the following probability:

$$
P\left(\mathbf{r}_{1}\right)=\exp \left(-\beta U\left(\mathbf{r}_{1}\right)\right) / w_{1},
$$

where

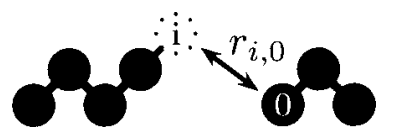

FIG. 2. Generation of a new trial site orientation during single rebridging of sites prior to chain closure.

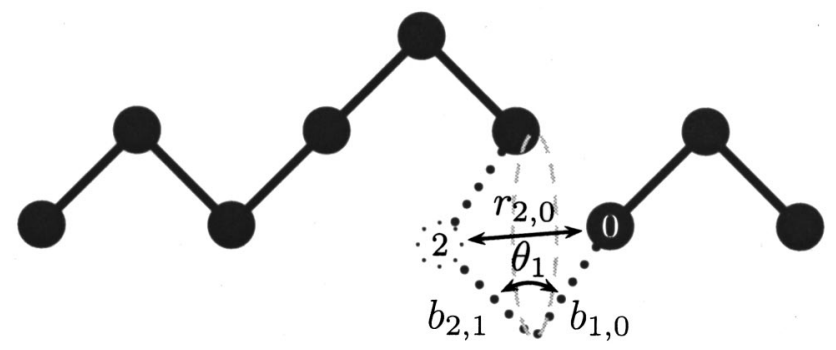

FIG. 3. Generation of a new trial site orientation for single rebridging for the last two sites.

$$
w_{1}=\sum_{j=1}^{N_{\text {samp }}} \exp \left(-\beta U\left(\mathbf{r}_{1(j)}\right)\right) .
$$

The overall probability of accepting a rebridging move of $N_{\text {site }}$ inner sites, where $N_{\text {site }}>2$, can be determined to be

$$
P_{\text {accept }}(\text { old } \rightarrow \text { new })=\min \left[1, \frac{W_{r b r}(\text { new })}{W_{r b r}(\text { old })}\right],
$$

where

$$
\begin{aligned}
& W_{r b r}(\text { new })=\frac{\Pi_{i=1}^{N_{\text {site }}} w_{i}(\text { new })}{J\left(\mathbf{r}_{2}\right)_{\text {new }} \Pi_{i=3}^{N_{\text {site }}} P_{\text {sep }}^{0}\left(r_{i, 0}\right)_{\text {new }}}, \\
& W_{r b r}(\text { old })=\frac{\Pi_{i=1}^{N_{\text {site }}} w_{i}(\text { old })}{J\left(\mathbf{r}_{2}\right)_{\text {old }} \Pi_{i=3}^{N_{\text {site }}} P_{\text {sep }}^{0}\left(r_{i, 0}\right)_{\text {old }}} .
\end{aligned}
$$

\section{DOUBLE-REBRIDGING TECHNIQUE}

The double-rebridging scheme proposed here utilizes the single-rebridging technique to simultaneously alter the connectivity of two linear polymer chains. A representation of the move is shown in Fig. 4. Note that the move preserves chain length and architecture. In principle, it can be performed by attempting to change the connectivity of a random inner section of two randomly selected chains. However, this approach exhibits a prohibitively low acceptance rate; a bias is therefore added for the selection of an attempted doublerebridging move. Before attempting the connectivity altering move, a double-bridging pair is selected according to

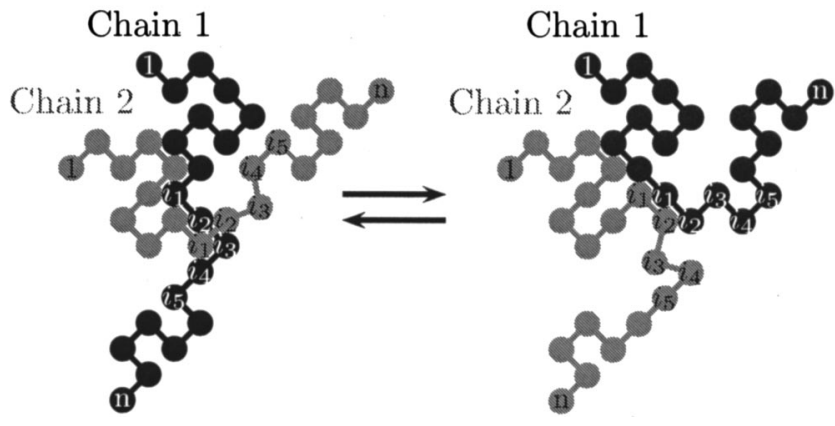

FIG. 4. Illustration of a double-rebridging move. The figure on the left is the original configuration. The figure on the right is the proposed new configuration. 


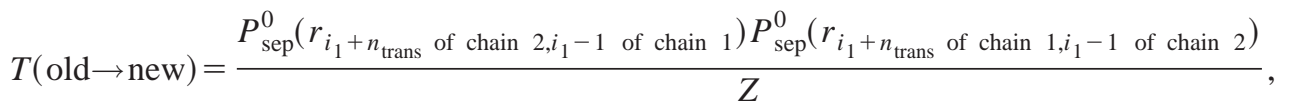

where $Z$ is defined as

$$
Z=\sum_{i=1}^{n_{\mathrm{chn}}-1} \sum_{j=i+1}^{n_{\mathrm{chn}}} \sum_{i_{s}=2}^{n-n_{\text {trans }}} P_{\text {sep }}^{0}\left(r_{i_{s}+n_{\text {trans }}} \text { of chain } j, i_{s}-1 \text { of chain } i\right) P_{\text {sep }}^{0}\left(r_{i_{s}+n_{\text {trans }} \text { of chain } i, i_{s}-1 \text { of chain } j}\right) \text {. }
$$

In Eqs. (10) and (11), $n_{\text {trans }}$ is the number of sites in a bridge, $i_{s}$ is the first site type of a given bridge, chain $i$ is the $i$ th chain in the system, $n_{\text {chn }}$ is the number of chains in the simulation cell, and $n$ is the number of sites in a chain. The summations in Eq. (11) are over all chain-length and architecture-preserving pairs. The selected double-bridging pair in Eq. (10) includes sites $i_{1}$ to $i_{1}+n_{\text {trans }}$ of chains 1 and 2.

The rebridging procedure is conducted as outlined in Sec. II. First, the rebridging weights are found for the two original bridges, $W_{r b r, 1}$ (old) and $W_{r b r, 2}($ old). Next, the two new connectivity altering bridges are constructed (with the order of bridge construction chosen at random), and the associated weights, $W_{r b r, 1}\left(\right.$ new) and $W_{r b r, 2}$ (new), are calculated. Finally, the probability of selecting the correct doublebridging pair to go from the new state to the old state, $T$ (new $\rightarrow$ old), is calculated with the new proposed configuration in the same manner as the calculation of $T$ (old $\rightarrow$ new). The acceptance probability for this move is given by

$$
\begin{aligned}
& P_{\text {accept }}(\text { old } \rightarrow \text { new }) \\
& \quad=\min \left[1, \frac{T(\text { new } \rightarrow \text { old }) W_{r b r, 1}(\text { new }) W_{r b r, 2}(\text { new })}{T(\text { old } \rightarrow \text { new }) W_{r b r, 1}(\text { old }) W_{r b r, 2}(\text { old })}\right] .
\end{aligned}
$$

For bookkeeping purposes, it is noted that the first labeled sites of a given chain remain with the given chain upon acceptance of a move. The higher labeled sites of the doublerebridging move are incorporated into the chain of the lower labeled sites upon acceptance of a move. This seemingly trivial point is essential when analyzing simulation results. It is essential that one of the chain ends does not change identity when analyzing end-to-end autocorrelation functions.

TABLE I. Bonding potential energy functions.

$$
\begin{aligned}
& \begin{array}{l}
\text { Bond stretching potential } \\
V(r) / k_{B}=\left(K_{r} / 2\right)\left(r-b_{e q}\right)^{2} \\
K_{r}=96500 \mathrm{~K} / \AA^{2} \\
\quad b_{\text {eq }}=1.54 \AA \quad(\mathrm{C}-\mathrm{C})
\end{array} \\
& V(\theta) / k_{B}=\left(K_{\theta} / 2\right)\left(\theta-\theta_{\mathrm{eq}}\right)^{2} \\
& \text { Bond bending potential } \\
& K_{\theta}=62500 \mathrm{~K} / \mathrm{rad}^{2} \\
& \theta_{\mathrm{eq}}=114.0^{\circ} \quad(\mathrm{C}-\mathrm{C}-\mathrm{C})
\end{aligned}
$$

Torsional potential

$V(\phi) / k_{B}=V_{0}+V_{1}(1+\cos \phi)+V_{2}(1-\cos 2 \phi)+V_{3}(1+\cos 3 \phi)$ Linear $(\mathrm{C}-\mathrm{C}-\mathrm{C}-\mathrm{C})$

$V_{0}=0$

$V_{2}=-68.19 \mathrm{~K}$

$V_{1}=355.04 \mathrm{~K}$

$V_{3}=791.32 \mathrm{~K}$

\section{SIMULATION FORCE FIELD}

All calculations presented here are for linear polyethylene melts at liquidlike densities. All simulations employ the NERD force field, ${ }^{14-16}$ in which united-atom sites represent $\mathrm{CH}_{3}$ and $\mathrm{CH}_{2}$ groups in linear polyethylene chains. Bonding interactions include bond stretching, bond angle bending, and torsional interactions due to rotations about bonds. Bond stretching and bond-angle bending are described by means of a harmonic potential, and a torsional potential of the form of Jorgensen et al. ${ }^{17}$ is used. A 12-6 Lennard-Jones potential energy function is adopted to describe site-site interactions for sites not involved in bonding interactions. For LennardJones interactions, we use Lorentz-Berthelot combining rules, which in previous work have been shown to be suitable for alkane mixtures. ${ }^{18}$ In all calculations, a cutoff radius of $10.0 \AA$ is employed for Lennard-Jones interactions, and standard tail corrections ${ }^{10,19}$ are implemented. A summary of the potential energy functions used in this work is provided in Tables I and II.

\section{SIMULATION CONDITIONS}

The systems considered in this work consist of linear polyethylene modeled as $\mathrm{C}_{200}$ and $\mathrm{C}_{1000}$ chains with $\mathrm{CH}_{3}$ end groups and $\mathrm{CH}_{2}$ middle groups. For the linear $\mathrm{C}_{200}$ case, a system of nine chains at $600 \mathrm{~K}$ and $0.716 \mathrm{~g} / \mathrm{cc}$ is studied. Canonical ensemble ( $N V T)$ simulations are performed using a conventional MD technique, an MC technique without double bridging, and an MC technique with double bridging. These systems represent polyethylene melts on the verge of entanglement.

A system of eight linear $\mathrm{C}_{1000}$ chains at $600 \mathrm{~K}$ and 1 bar is also simulated. For this polyethylene model, the chains are above the entanglement length. For this case, isothermalisobaric $(N P T)$ simulations are conducted with an MD technique, an $\mathrm{MC}$ technique without double bridging, and an $\mathrm{MC}$ technique with double bridging. All simulations are conducted on a pool of computers which perform floating point operations at the rate of 0.3-0.7 GFlops.

TABLE II. Nonbonding potential energy functions.

Lennard-Jones interaction potential:

$V(r) / k_{B}=4 \epsilon\left[(\sigma / r)^{12}-(\sigma / r)^{6}\right]$

$\sigma_{\mathrm{CH}_{2}\left(s p^{3}\right)}=3.93 \AA$

Polyethylene chains

$\sigma_{\mathrm{CH}_{3}\left(s p^{3}\right)}=3.91 \AA$

$\epsilon_{\mathrm{CH}_{2}\left(s p^{3}\right)}=45.8 \mathrm{~K}$

$\epsilon_{\mathrm{CH}_{3}\left(s p^{3}\right)}=104.0 \mathrm{~K}$ 
TABLE III. Simulation conditions for linear $\mathrm{C}_{200}$ and $\mathrm{C}_{1000}$ simulations. The CPU requirements are shown for all cases. The proportion of individual moves is also indicated for the Monte Carlo simulations.

\begin{tabular}{|c|c|c|c|c|c|c|c|}
\hline Simulation & $\begin{array}{c}\text { Varying } \\
\text { volume } \\
\text { hybrid \% }\end{array}$ & $\begin{array}{c}\text { Constant } \\
\text { volume } \\
\text { hybrid \% }\end{array}$ & $\begin{array}{l}\text { End-site } \\
\text { CCB \% }\end{array}$ & $\begin{array}{l}\text { Reptation } \\
\text { CCB \% }\end{array}$ & $\begin{array}{l}\text { Inner-chain } \\
\text { rebridging \% }\end{array}$ & $\begin{array}{c}\text { Double } \\
\text { rebridging \% }\end{array}$ & $\begin{array}{c}\text { CPU time } \\
\text { per } 10^{6} \text { steps } \\
\text { [days] }\end{array}$ \\
\hline \multicolumn{8}{|l|}{$\mathrm{C}_{200}$ systems } \\
\hline$N V T-\mathrm{MD}$ & $\ldots$ & $\ldots$ & $\ldots$ & $\ldots$ & $\ldots$ & $\ldots$ & 5.25 \\
\hline $\begin{array}{l}N V T \text {-MC without } \\
\text { double rebridging }\end{array}$ & $\cdots$ & $\cdots$ & 10 & 20 & 70 & $\cdots$ & 1.07 \\
\hline $\begin{array}{l}N V T \text {-MC with } \\
\text { double rebridging }\end{array}$ & $\cdots$ & $\cdots$ & 5 & 10 & 35 & 50 & 2.33 \\
\hline \multicolumn{8}{|l|}{$\mathrm{C}_{1000}$ Systems } \\
\hline$N P T-\mathrm{MD}$ & $\cdots$ & $\cdots$ & $\cdots$ & $\cdots$ & $\cdots$ & $\cdots$ & 9.67 \\
\hline $\begin{array}{l}N P T \text {-MC without } \\
\text { double rebridging }\end{array}$ & 0.8 & 0.2 & 10 & 19 & 70 & $\cdots$ & 2.20 \\
\hline $\begin{array}{l}N P T-\mathrm{MC} \text { with } \\
\text { double rebridging }\end{array}$ & 0.4 & 0.1 & 5 & 9.5 & 35 & 50 & 3.27 \\
\hline
\end{tabular}

\section{A. Molecular dynamics simulations}

For the linear $\mathrm{C}_{200}$ case, canonical molecular dynamics $(N V T-\mathrm{MD})$ simulations are conducted using a Nosé-Hoover ${ }^{20-23}$ scheme with a time step, $d t^{*}$ $=0.001(0.0016 \mathrm{ps})$ in reduced Lennard-Jones units. The time step is reduced in terms of $\sigma_{\mathrm{CH}_{2}} \epsilon_{\mathrm{CH}_{2}}$, and the mass of the $\mathrm{CH}_{2}$ sites.

For the linear $\mathrm{C}_{1000}$ case, isothermal-isobaric molecular dynamics (NPT-MD) simulations are conducted using a Berendsen weak-coupling scheme ${ }^{24,25}$ with a reduced time step, $d t^{*}$, of 0.001 (0.0016 ps). The reduced pressure coupling parameter, $\tau_{P}^{*}$, is set to $4.2(6.8 \mathrm{ps})$ and the reduced temperature coupling parameter, $\tau_{T}^{*}$, is set to $0.001(0.0016 \mathrm{ps})$.

The CPU requirements for the two different molecular dynamics cases can be found in Table III.

\section{B. Conventional Monte Carlo simulations}

For conventional Monte Carlo simulations, inner-chain rebridging, ${ }^{8}$ end-site continuum configurational bias (CCB) ${ }^{26,27} \mathrm{CCB}$ reptation, and hybrid Monte Carlo moves are used.

For all Monte Carlo simulations, single-rebridging moves are carried out for 2-6 sites per move and ten trial orientations generated per site. The acceptance of this move is on the order of $6 \%$. End-site CCB moves are conducted for 1-6 polyolefin end sites per move, and ten trial orientations per site. The acceptance is on the order of $45 \%$. CCB reptation moves are performed for 1-4 sites per move and ten trial orientations generated per site. The acceptance of this move is on the order of $20 \%$.

For NPT Monte Carlo simulations, constant and varying volume hybrid Monte Carlo simulations are also implemented. In the constant volume hybrid- $N V T$ procedure, ${ }^{28,29}$ five molecular dynamics steps are used to generate a global trial Monte Carlo move with a reduced time step of 0.001 $(0.0016 \mathrm{ps})$. The acceptance of this move is on the order of $71 \%$. In the reversible Berendsen hybrid-NPT procedure ${ }^{24,25,30}$ five molecular dynamics steps are used to generate a global trial Monte Carlo move with a reduced time step of 0.001 ( $0.0016 \mathrm{ps})$. The acceptance of this move is on the order of $41 \%$.

The proportion of moves and CPU requirements for the conventional $\mathrm{C}_{200}$ and $\mathrm{C}_{1000}$ Monte Carlo simulations (MC simulations without double rebridging) are shown in Table III.

\section{Double-rebridging Monte Carlo simulations}

To test the proposed double-rebridging scheme, MC simulations with $50 \%$ double-rebridging moves are also performed. The other trial moves are conducted in the same proportion as in the conventional MC simulations. The acceptance of non-double-rebridging moves is similar to that of the "standard" MC simulations. The proportion of moves for the $\mathrm{C}_{200}$ and $\mathrm{C}_{1000}$ Monte Carlo simulations with double rebridging is shown in Table III.

The double-rebridging moves are performed for five site bridges with 20 trial orientations generated per site; five site bridges are used based on the optimal performance as compared to longer and shorter bridges observed during short simulations with the $\mathrm{C}_{1000}$ system. The acceptance of the double-rebridging moves is on the order of $0.02 \%$, i.e., one accepted move for every 5000 attempts.

TABLE IV. Simulation characteristics for various double-rebridging moves. Simulations are for systems of eight linear $\mathrm{C}_{1000}$ chains at $600 \mathrm{~K}$ and 1 bar. Simulations are conducted with moves indicated in Table III and run for 5 $\times 10^{6}$ steps (10-30 days) on a pool of computers, all of which perform floating-point operations at the rate of 0.6-0.7 GFlops.

\begin{tabular}{cccc}
\hline \hline $\begin{array}{c}\text { Number of sites } \\
\text { per bridge }\end{array}$ & $\begin{array}{c}\text { Acceptance } \\
{[\%]}\end{array}$ & $\begin{array}{c}\text { CPU time per } \\
\text { double-rebridging } \\
\text { move [s] }\end{array}$ & $\begin{array}{c}\text { Average number of } \\
\text { bridgeable pairs } \\
\text { per move }\end{array}$ \\
\hline 3 & 0.037 & 0.33 & 9.3 \\
5 & 0.021 & 0.48 & 75 \\
7 & 0.012 & 0.62 & 184 \\
9 & 0.0035 & 0.74 & 588 \\
11 & 0.0012 & 0.90 & 965 \\
\hline \hline
\end{tabular}




\section{RESULTS AND DISCUSSION}

\section{A. Double-rebridging optimization}

Table IV shows the acceptance rate, double-rebridging move CPU requirement, and average number of "bridgeable" pairs for double-rebridging simulations with 3-11 site bridges. In the nomenclature of Sec. III, the number of bridgeable pairs is the number of proposed new doublebridging pairs with $i 1-1$ and $i 1+n_{\text {trans }}$ separated by less than $b_{\text {eq }}\left(n_{\text {trans }}+1\right)$ for both new bridges. In other words, it is the number of pairs that, on average, could be geometrically connected without altering the architecture of the molecules. From Table IV, it is apparent that the acceptance increases and the $\mathrm{CPU}$ requirement decreases for shorter bridges. However, the average number of bridgeable pairs drastically decreases with shorter bridges. This indicates that the number of possibilities to form short bridges is small. This limited number of possibilities will greatly reduce the sampling space for this type of move.

The relative effectiveness is determined based on the initial decay of the chain end-to-end site vector $\left(\mathbf{R}_{0}\right)$ autocorrelation function. The results are shown in Fig. 5. From Fig. 5, it is seen that all double-rebridging cases are superior to the case without double rebridging in the relaxation of the end-to-end site vector autocorrelation function. It is also seen that the case of five site bridges provides the fastest relaxation for the simulation conditions given in Table III. Therefore, five site bridges are used for the rest of the calculations.

\section{B. Linear polyethylene results}

Figure 6 shows the initial decay of the chain end-to-end site vector autocorrelation function for the linear $\mathrm{C}_{200}$ melt at $600 \mathrm{~K}$ and $0.716 \mathrm{~g} / \mathrm{cc}$ using the three simulation techniques considered here as a function of CPU time. As apparent in

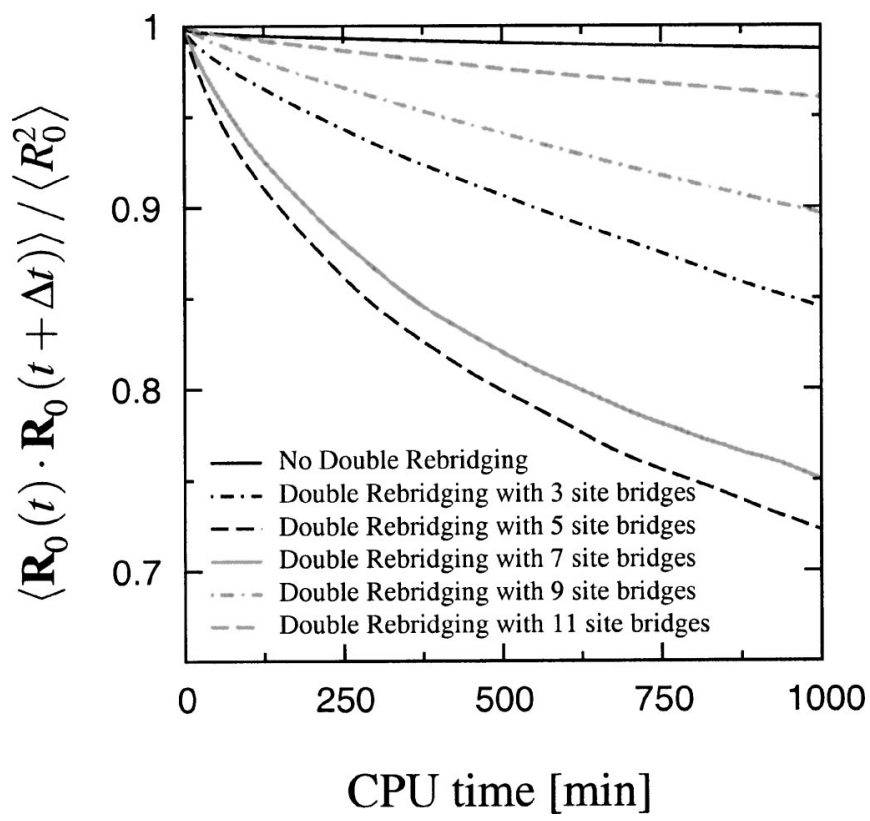

FIG. 5. Initial decay of the end-to-end vector autocorrelation functions for systems of eight linear $\mathrm{C}_{1000}$ chains at $600 \mathrm{~K}$ and 1 bar. Details for these Monte Carlo simulations are provided in Tables III and IV.

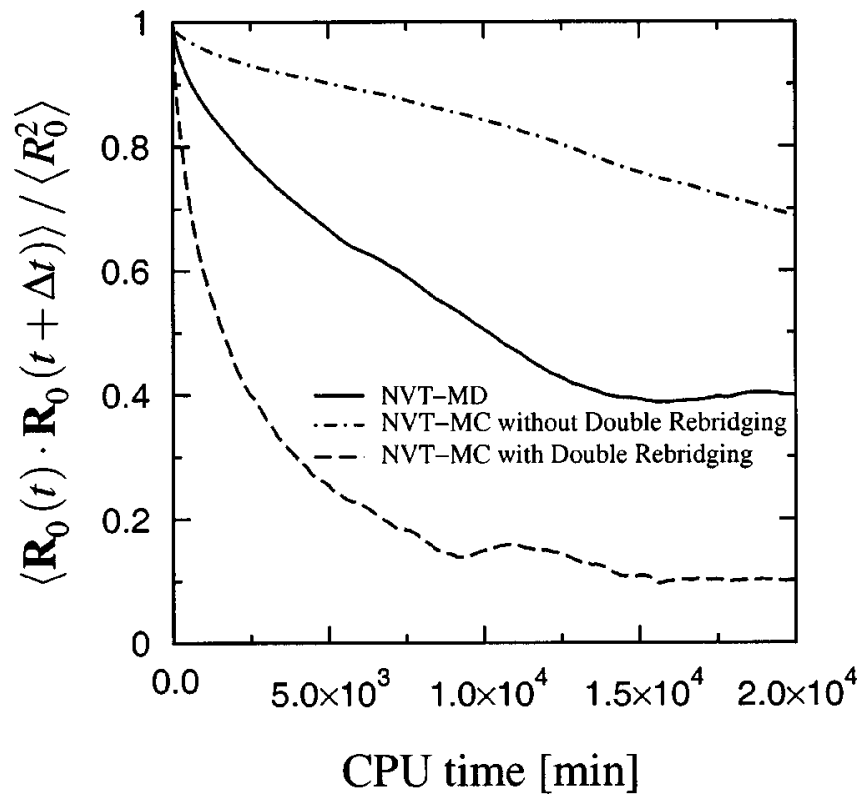

FIG. 6. End-to-end vector autocorrelation functions for three $N V T$ simulations of a system of nine linear $\mathrm{C}_{200}$ chains at $600 \mathrm{~K}$ and $0.716 \mathrm{~g} / \mathrm{cc}$. Details for these Monte Carlo simulations are provided in Table III.

Fig. 6, the end-to-end vector autocorrelation function relaxes more rapidly for the simulation that includes the doublerebridging technique. The speed-up as compared to the standard MC is several orders of magnitude.

Figure 7 shows the initial decay of the chain end-to-end site vector autocorrelation function for the three linear $\mathrm{C}_{1000}$ simulations as a function of CPU time. The doublerebridging scheme is again superior to the standard $\mathrm{MC}$ and MD techniques for the relaxation of the end-to-end vector. The enhancement is larger for this system than for the $C_{200}$ system. Since the polyethylene model considered here is above the entanglement length, the end-to-end vector is ex-

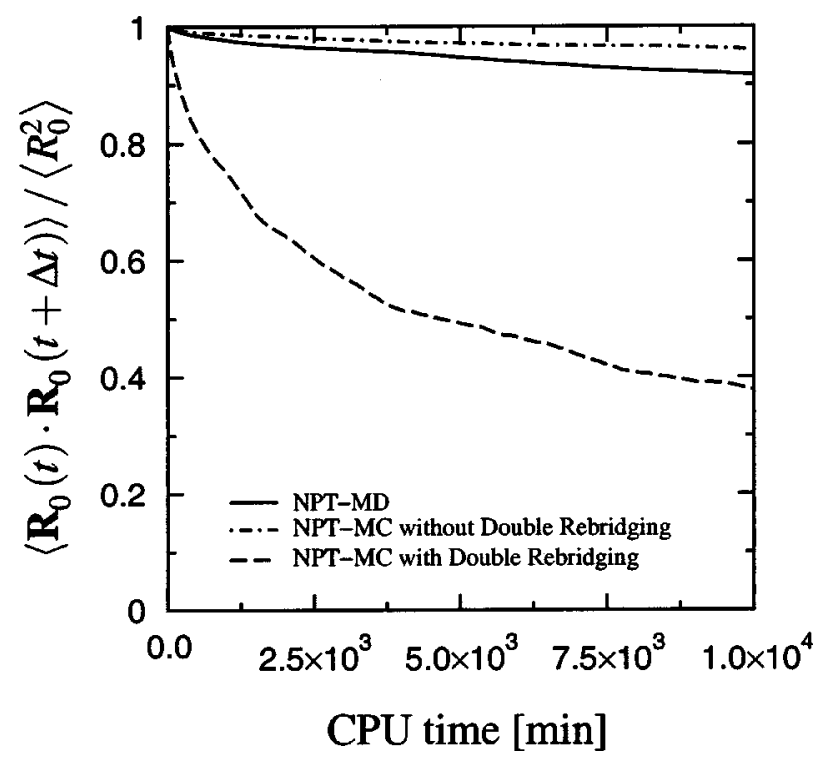

FIG. 7. End-to-end vector autocorrelation functions for three NPT simulations of a system of eight linear $\mathrm{C}_{1000}$ chains at $600 \mathrm{~K}$ and 1 bar. Details for these Monte Carlo simulations are provided in Table III. 
TABLE V. Density and structural property results for linear polyethylene at $600 \mathrm{~K}$ and 1 bar. The simulation results are for the polyethylene melt modeled as a system of eight linear $\mathrm{C}_{1000}$ chains. The experimental density results are extrapolated from the results of Walsh et al. (Ref. 31). The experimental $C_{\infty}$ results are extrapolated from data at 413-440 K (Refs. 32-34).

\begin{tabular}{llcccc}
\hline \hline & Density $[\mathrm{g} / \mathrm{cc}]$ & $C_{\infty}$ & $\left\langle R_{0}^{2}\right\rangle /\left\langle R_{g}^{2}\right\rangle$ & $l_{\text {Kuhn }}[\AA]$ & $n / n_{\text {Kuhn }}$ \\
\hline Simulation & & & & & \\
$\quad N P T$-MD & $0.685 \pm 0.003$ & $6.1 \pm 1.9$ & 8.27 & 9.36 & 6.09 \\
$\quad N P T$-MC without double rebridging & $0.695 \pm 0.015$ & $5.2 \pm 1.6$ & 8.86 & 8.04 & 5.23 \\
$\quad N P T$-MC with double rebridging & $0.690 \pm 0.010$ & $5.9 \pm 0.4$ & 6.68 & 8.26 & 5.37 \\
Experiment (Refs. 31-34) & 0.685 & $6.0 \pm 0.3$ & $\cdots$ & $\ldots$ & $\ldots$ \\
\hline \hline
\end{tabular}

tremely slow to relax with MD and conventional MC methods. Upon comparison with the $\mathrm{C}_{200}$ simulations, the relaxation of the end-to-end vector for the MC double-rebridging simulation appears to be relatively insensitive to chain length.

The density and characteristic ratio $\left(C_{\infty} \equiv\left\langle R_{0}^{2}\right\rangle / n l^{2}\right)$ are calculated for each simulation condition and compared with experimental data. Furthermore, $\left\langle R_{0}^{2}\right\rangle /\left\langle R_{g}^{2}\right\rangle\left(R_{g}\right.$ is the radius of gyration), the Kuhn length $\left(l_{\text {Kuhn }}\right)$, and number of carbon bonds per Kuhn bond $\left(n / n_{\text {Kuhn }}\right)$ are calculated for each simulation condtion. The Kuhn length, $l_{\text {Kuhn }}$, is defined as $\left\langle R_{0}^{2}\right\rangle / L_{c}$, where $L_{c}$ is the contour length of a chain. The number of Kuhn bonds is defined so that $\left\langle R_{0}^{2}\right\rangle=n_{\text {Kuhn }} l_{\text {Kuhn }}^{2}$ (i.e., Kuhn segments behave like a freely jointed chain). The results are presented in Table V.

Satisfactory agreement is observed between simulated and experimental densities for all simulations. In addition, agreement is found between simulated and experimental $C_{\infty}$ for all three simulation methods. However, the MC with double-rebridging technique yields much smaller error in $C_{\infty}$ as compared to the other two simulation methods. Note that $\left\langle R_{0}^{2}\right\rangle /\left\langle R_{g}^{2}\right\rangle$ is equal to 6 for fully flexible chains under theta conditions and equal to 12 for rigid rods. It is not expected that this system should differ too much from the fully flexible chain model. With this in mind, the simulated $\left\langle R_{0}^{2}\right\rangle /\left\langle R_{g}^{2}\right\rangle$ appears to be more realistic for the MC with doublerebridging technique than the simulated $\left\langle R_{0}^{2}\right\rangle /\left\langle R_{g}^{2}\right\rangle$ values from the other two simulation methods. However, experimental data for comparison are not available.

TABLE VI. Effect of system size on the performance of the doublerebridging move. All simulations are conducted at $600 \mathrm{~K}$ and 1 bar with polyethylene modeled as linear $C_{1000}$. Simulations are conducted using five site bridges for the double-rebridging move and with moves in proportion to those of Table III. All simulations are run for $5 \times 10^{6}$ steps $(\sim 15$ days $)$ on a pool of computers, all of which perform floating-point operations at the rate of $0.6-0.7$ GFlops.

\begin{tabular}{ccc}
\hline \hline $\begin{array}{c}\text { Number of } C_{1000} \\
\text { chains in the system }\end{array}$ & $\begin{array}{c}\text { Acceptance } \\
{[\%]}\end{array}$ & $\begin{array}{c}\text { Average number of } \\
\text { bridgeable pairs } \\
\text { per move }\end{array}$ \\
\hline 2 & 0.011 & 25 \\
4 & 0.016 & 46 \\
8 & 0.021 & 75 \\
12 & 0.025 & 95 \\
\hline \hline
\end{tabular}

\section{Effect of system size on double-rebridging performance}

To illustrate the effect of system size, the performance of the double-rebridging technique is examined for systems of 2-12 linear $C_{1000}$ chains. All simulations are conducted at $600 \mathrm{~K}$ and 1 bar, with five site bridges for the doublerebridging move and with the MC moves prescribed in Sec. V C. Table VI shows the acceptance rate and average number of bridgeable pairs for the different cases. As is evident from Table VI, the acceptance of the double-rebridging move increases with system size as the number of bridgeable pairs increases.

\section{CONCLUSIONS}

A new variable connectivity double-rebridging MC technique has been developed. It is seen to dramatically speed up the relaxation of the end-to-end vector for entangled linear polyethylenes of 200 and 1000 carbons. The relaxation of the end-to-end vector appears to be independent of chain length with this new technique. This new technique is proposed as a simple and flexible alternative to a recently proposed variable connectivity double-rebridging technique. ${ }^{1}$ The calculated characteristic ratios, $C_{\infty}$, from simulations involving the new technique are consistent with experimental results. Additionally, $\left\langle R_{0}^{2}\right\rangle /\left\langle R_{g}^{2}\right\rangle$ and Kuhn parameters are calculated with the new technique.

Future simulations will be conducted at conditions of experimental and industrial interest. Parallel tempering techniques ${ }^{35-37}$ will also be used in these cases to further enhance the simulations. In addition to linear polyethylene, simulations of branched polyolefins are of tremendous experimental and industrial interest. The variable connectivity method of this study can be expanded to encompass branched systems in a straightforward manner; this will be addressed in the future.

\section{ACKNOWLEDGMENT}

Financial support from the U.S. Department of Energy (DE-FG02-99ER14961) is gratefully acknowledged.

\footnotetext{
${ }^{1}$ N. Ch. Karayiannis, V. G. Mavrantzas, and D. N. Theodorou, Phys. Rev. Lett. 88, 105503 (2002).

${ }^{2}$ V. G. Mavrantzas, T. D. Boone, E. Zervopoulou, and D. N. Theodorou, Macromolecules 32, 5072 (1999).
} 
${ }^{3}$ P. V. K. Pant and D. N. Theodorou, Macromolecules 28, 7224 (1995).

${ }^{4}$ A. Uhlherr, V. G. Mavrantzas, M. Doxastakis, and D. N. Theodorou, Macromolecules 34, 8554 (2001).

${ }^{5}$ M. Doxastakis, V. Mavrantzas, and D. Theodorou, J. Chem. Phys. 115, 11339 (2001).

${ }^{6}$ M. Doxastakis, V. Mavrantzas, and D. Theodorou, J. Chem. Phys. 115, 11352 (2001).

${ }^{7}$ A. Uhlherr, M. Doxastakis, V. Mavrantzas, D. Theodorou, S. J. Leak, N. E. Adam, and P. E. Nyberg, Europhys. Lett. 57, 506 (2002).

${ }^{8}$ Z. Chen and F. A. Escobedo, J. Chem. Phys. 113, 11382 (2000).

${ }^{9}$ B. Smit and J. I. Siepmann, J. Phys. Chem. 98, 8442 (1994).

${ }^{10}$ D. Frenkel and B. Smit, Understanding Molecular Simulation: From Algorithms to Applications (Academic, New York, 1996).

${ }^{11}$ G. Marsaglia, Ann. Math. Stat. 43, 645 (1972).

${ }^{12}$ F. A. Escobedo and J. J. de Pablo, J. Chem. Phys. 102, 2636 (1995).

${ }^{13}$ F. A. Escobedo and J. J. de Pablo, J. Chem. Phys. 104, 4788 (1996).

${ }^{14}$ S. K. Nath, F. A. Escobedo, and J. J. de Pablo, J. Chem. Phys. 108, 9905 (1998).

${ }^{15}$ S. K. Nath and J. J. de Pablo, Mol. Phys. 98, 231 (2000).

${ }^{16}$ S. K. Nath, B. J. Banaszak, and J. J. de Pablo, J. Chem. Phys. 114, 3612 (2001).

${ }^{17}$ W. L. Jorgensen, J. D. Madura, and C. J. Swensen, J. Am. Chem. Soc. 106, 6638 (1984).

${ }^{18}$ S. K. Nath, F. A. Escobedo, J. J. de Pablo, and I. Patramai, Ind. Eng. Chem. Res. 37, 3195 (1998).

${ }^{19}$ M. P. Allen and D. J. Tildesley, Computer Simulation of Liquids (Oxford University Press, New York, 1987).
${ }^{20}$ S. Nosé, Mol. Phys. 52, 255 (1984).

${ }^{21}$ S. Nosé, J. Chem. Phys. 81, 511 (1984).

${ }^{22}$ W. G. Hoover, Phys. Rev. A 31, 1695 (1985).

${ }^{23}$ G. J. Martyna, D. J. Tobias, and M. L. Klein, J. Chem. Phys. 101, 4177 (1994).

${ }^{24}$ H. J. C. Berendsen, J. P. M. Postma, W. F. Gunsteren, A. DiNola, and J. R. Haak, J. Chem. Phys. 81, 3684 (1984).

${ }^{25}$ W. F. Gunsteren and H. J. C. Berendsen, Angew. Chem., Int. Ed. Engl. 29, 992 (1990).

${ }^{26}$ M. Laso, J. J. de Pablo, and U. W. Suter, J. Chem. Phys. 97, 2817 (1992).

${ }^{27}$ J. I. Siepmann and D. Frenkel, Mol. Phys. 75, 59 (1992).

${ }^{28}$ S. Duane, A. D. Kennedy, B. J. Pendleton, and D. Roweth, Phys. Lett. B 195, 216 (1987).

${ }^{29}$ S. K. Nath, J. J. de Pablo, and A. D. DeBellis, J. Am. Chem. Soc. 121, 4252 (1999)

${ }^{30}$ R. Faller and J. J. de Pablo, J. Chem. Phys. 116, 55 (2002).

${ }^{31}$ G. T. Dee, T. Ougizawa, and D. J. Walsh, Polymer 33, 3462 (1992).

${ }^{32}$ L. J. Fetters, D. J. Lohse, D. Richter, T. A. Witten, and A. Zirkel, Macromolecules 27, 4639 (1994).

${ }^{33}$ L. J. Fetters, D. J. Lohse, S. T. Milner, and W. M. Graessley, Macromolecules 32, 6847 (1999).

${ }^{34}$ J. C. Horton, G. L. Squires, A. T. Boothroyd, L. J. Fetters, A. R. Rennie, C. J. Glinka, and R. A. Robinson, Macromolecules 22, 681 (1989).

${ }^{35}$ U. H. E. Hansmann, Chem. Phys. Lett. 281, 140 (1997).

${ }^{36}$ Q. Yan and J. J. de Pablo, J. Chem. Phys. 111, 9509 (1999).

${ }^{37}$ Q. Yan and J. J. de Pablo, J. Chem. Phys. 113, 1276 (2000). 